\title{
On Volterra-type integral equations in noncompact metric space
}

\author{
Andrey Zahariev*, Atanaska Georgieva and Lozanka Trenkova
}

"Correspondence:

zandrey@uni-plovdiv.bg

Faculty of Mathematics and

Informatics, University of Plovdiv,

Plovdiv, 4000, Bulgaria

\begin{abstract}
In the present work, we consider one of the possible generalizations of linear and nonlinear Volterra integral equations of the second kind in the case when the independent variable belongs to an arbitrary noncompact metric space. Sufficient conditions are obtained for the existence of solutions of Volterra-type integral equations in the nonhomogeneous case. Some applications of the obtained results to the integral inequalities are given.
\end{abstract}

MSC: 35Q99; 35R35; 65M12;65M70

Keywords: Volterra-type integral equations; metric space; Banach space; integral inequalities

\section{Introduction}

Integral equations and inequalities have been of considerable significance in mathematics and have held a central place in the attention of mathematicians during the last few decades. In the past few years, integral equations have proved to be of tremendous use in several applied fields, such as population dynamics, spread of epidemics, automatic control theory, network theory and the dynamics of nuclear reactors. The main application of the integral inequalities is that they provide an explicit bounds of the unknown functions, which are a very useful and important device in the study of many qualitative as well as quantitative properties of solutions of nonlinear differential equations. In the theory of integral inequalities, an enormous amount of effort has been devoted to the polishing of classical approaches to proving the inequalities. The techniques of these proofs, in general based on the classical mathematical analysis, lead up to virtuosity and significantly depend on the number of independent variables and the geometry of the domain of integration. The mathematical literature provides a good deal of information about the integral equations and inequalities and an excellent amount of results may be found in the monographs [1-4] and the comprehensive list of references therein. We can find several different directions and approaches to this field of study in [5, 6] and [7]. All monographs mentioned above are proposed because the authors have an essential contribution to the theory presented in their books.

The aim of this paper is to develop the approach introduced for the linear case in the work [8] for the nonlinear one. The main idea introduced in [8] is simple - establishing conditions under which the unique solution of equation (2.1) is an upper bound of all solutions of inequality (2.2). A similar idea was used in [9] too. In view of the applications,

(O2014 Zahariev et al.: licensee Springer. This is an Open Access article distributed under the terms of the Creative Commons Attribution License (http://creativecommons.org/licenses/by/2.0), which permits unrestricted use, distribution, and reproduction in any medium, provided the original work is properly cited. 
it is important to study also in which cases, as in the one-dimensional case, the exponential function is a sharp estimation for the Gronwall-type multidimensional inequalities. In the case when the domains of integration are the Cartesian product of bounded and closed intervals this is true (see [10]), but some results and examples given in [8] indicate that the answer of this problem generally speaking is negative, even in the linear case. Moreover, the type of the sharp estimation function will depend on the geometry of the domains of integration in the high dimensional cases.

The paper is organized as follows. In Section 2 we introduce an abstract nonlinear analogue of the Volterra equations and its corresponding inequality. In Section 3 we discuss the possibility to replace without loss of generality one compact domain of integration with another one which is close to it in the measure and metric sense, but have better properties. Section 4 is devoted to the study of Volterra equation (2.1) introduced below. Section 5 includes the results concerning the integral inequality (2.2), and in Section 6 some applications of our results obtained in the previous sections are given as well as examples illustrating the applications are presented.

We note that different kinds of results used in the conceptual plan of our approach introduced in [8] are received in [11-14] and some interesting ideas in the atomic case are developed in [15] and [16].

\section{Preliminaries}

Let $\Omega$ be a complete metric space with metric $\rho$, let $B_{\Omega} \subset 2^{\Omega}$ denote the $\sigma$-algebra of the Borel subsets of $\Omega$, and let $\mu: B_{\Omega} \rightarrow[0, \infty)$ be a nontrivial $\sigma$-finite Borel measure. We will denote by $U(x, \varepsilon)=\{y \in \Omega \mid \rho(x, y)<\varepsilon\}$ the open balls with a center point $x \in \Omega$, and radius $\varepsilon>0$. If $G$ is an arbitrary subset of $\Omega$, then $\partial G$ denotes the boundary of $G$ and $U(G, \varepsilon)$ denotes the $\varepsilon$-neighborhood of $G$.

Let $B$ be a real Banach space with the norm $\|\cdot\|_{B}$, and let $V \subset B$ be a cone in $B$. Then we can introduce a partial ordering in $B$ associated with the cone $V$, i.e., $u \geq v$ when $u-v \in V$. We shall write $u>v$ to indicate that $u-v \in V$, but $u \neq v$. Denote by $C(G, B)$, $G \subset \Omega$ is an arbitrary compact subset, the Banach space of all continuous maps $f: G \rightarrow B$ with the norm $\|f\|_{G}=\sup _{y \in G}\|f(y)\|_{B}$, by $C_{b}(\Omega, B)$ the Banach space of all bounded continuous maps $f: \Omega \rightarrow B$ with the norm $\|f\|=\sup _{y \in \Omega}\|f(y)\|_{B}$ and by $C(\Omega, B)$ the linear topological space of all continuous maps $f: \Omega \rightarrow B\left(\lim _{n \rightarrow \infty} f_{n}=f_{0}\right.$ if for each $x \in \Omega$ we have $\left.\lim _{n \rightarrow \infty}\left\|f_{n}(x)-f_{0}(x)\right\|=0\right)$.

Let $G, H \subset \Omega$ be arbitrary. We will denote by $G, H \subset \Omega$ with

$$
G \Delta H=(G \backslash H) \cup(H \backslash G)
$$

the symmetric difference of the sets $G$ and $H$.

Definition 2.1 A set $G \in B_{\Omega}$ is called an atom for the measure $\mu$ if $\mu(G)>0$, and for each $H \in B_{\Omega}$ with $\mu(G)>\mu(H)$ one has $\mu(H)=0$.

Partially, if for some point $x \in \Omega$ we have that $\mu(\{x\})>0$, then we say that this point is an atom for the measure $\mu$; otherwise, we say that the point is nonatomic.

We introduce the map $M: \Omega \rightarrow 2^{\Omega}$ which associates every point $x \in \Omega$ with a subset $M_{x} \subset \Omega$. Generally speaking it is not obligatory for the point $x \in \Omega$ to be an element of $M_{x}$. 
Consider the equation

$$
f(x)=p(x)+\int_{M_{x}} Q(x, y, f(y)) d \mu_{y}
$$

and the inequality

$$
g(x) \leq p(x)+\int_{M_{x}} Q(x, y, g(y)) d \mu_{y}
$$

where the operator $Q: \Omega \times \Omega \times B \rightarrow B$ and $f, g, p \in C\left(M_{x}, B\right), x \in \Omega$.

Following [8] we will say that conditions (A) hold if for the map $M: \Omega \rightarrow 2^{\Omega}$ the following conditions are fulfilled:

(A1) For every point $x \in \Omega$, the set $M_{x}$ is compact.

(A2) For each $\varepsilon>0$ and every $x \in \Omega$, there exists $\delta>0$ such that, for each $y \in \Omega$ with $\rho(y, x)<\delta$, we have that $\mu\left(M_{x} \Delta M_{y}\right)<\varepsilon$.

(A3) For every $x \in \Omega$, the inclusion $M_{y} \subseteq M_{x}$ holds for each $y \in M_{x}$.

(A4) There exists $x_{0} \in \Omega$ such that $\mu\left(M_{x_{0}}\right)=0$.

For every map $M: \Omega \rightarrow 2^{\Omega}$ for which conditions (A) hold, we will define $\mathbf{M}=\left\{M_{x} \mid x \in\right.$ $\Omega\}$.

Remark 2.2 Generally speaking it is not necessary for the set $M_{x}$ to include the point $x$. A simple example when this is true is $\Omega=[0, \infty)$ and $M_{x}=\left[0, \frac{x}{2}\right]$.

If the operator $Q$ is continuous for every $x \in \Omega$ in the set $M_{x} \times M_{x} \times B$, then the integral in (2.1) and (2.2) exists; see, e.g., $[17,18]$.

\section{Domains of integration}

It is well known that even for finite dimensional metric spaces with Lebesgue measure two compact subsets in them can be very close in the measure sense (partially, having equal measures) and at the same time very far in the metric sense (having very different diameters). From this fact it follows that using as domains of integration arbitrary compact sets cannot be convenient enough in lots of cases. It is easy to see that generally this is true even for the compact sets belonging to the set $\mathbf{M}$. The aim of this section is to find some other set $\mathbf{M}^{\mu} \subset \mathbf{2}^{\Omega}$, whose elements have better properties. Since all these sets will be used as domains of integration, the best result will be if for every element $M_{x} \in \mathbf{M}$ we can find an element $M_{x}^{\mu} \in \mathbf{M}^{\mu}$ such that $\mu\left(M_{x}^{\mu} \Delta M_{x}\right)=0$, and both elements are close in the metric sense too.

Let $G \subset B_{\Omega}$ be an arbitrary set.

Definition 3.1 The nonatomic point $x \in \Omega$ will be called essential for the set $G$ if for each $\varepsilon>0$ we have $\mu(G \cap U(x, \varepsilon))>0$.

Definition 3.2 The nonatomic point $x \in \Omega$ will be called unessential for the set $G$ if there exists $\varepsilon_{x}>0$ such that $\mu\left(U\left(x, \varepsilon_{x}\right) \cap G\right)=0$.

Let us consider the points $x \in \partial G$.

Lemma 3.3 For every point $x \in \partial G$ which is unessential for $G$, there exists $\varepsilon_{x}>0$ such that the open ball $U\left(x, \varepsilon_{x}\right)$ does not include essential points for $G$. 
Proof Let the point $x \in \partial G$ be an unessential for $G$. Then there exists $\varepsilon_{x}>0$ such that $\mu\left(U\left(x, \varepsilon_{x}\right) \cap G\right)=0$. Assume that there exists an essential for $G$ point $z_{x} \in U\left(x, \varepsilon_{x}\right)$. Then there exists a number $\varepsilon_{z}<\varepsilon_{x}$ such that $U\left(z_{x}, \varepsilon_{z}\right) \subset U\left(x, \varepsilon_{x}\right)$, and $\mu\left(U\left(z_{x}, \varepsilon_{z}\right) \cap G\right)>0$ and therefore

$$
\mu\left(U\left(x, \varepsilon_{x}\right) \cap G\right) \geq \mu\left(U\left(z_{x}, \varepsilon_{z}\right) \cap G\right)>0,
$$

which is impossible.

Corollary 3.4 Every isolated point $x \in \partial G$ is either unessential for $G$, or an atom for the measure $\mu$.

Proof Let $G \in B_{\Omega}$ be an arbitrary set and $x_{0} \in G$ be an isolated point. Then there exists $r>0$ such that $G \cap U\left(x_{0}, r\right)=\left\{x_{0}\right\}$ and therefore

$$
\mu\left(G \cap U\left(x_{0}, r\right)\right)=\mu\left(\left\{x_{0}\right\}\right) .
$$

Then either $\mu\left(\left\{x_{0}\right\}\right)>0$ and $x_{0}$ is an atom, or $\mu\left(\left\{x_{0}\right\}\right)=0$ and then $x_{0}$ is an unessential point.

Corollary 3.5 The nonatomic point $x \in \partial G$ is essential for $G$ if for each $\varepsilon>0$ in the open ball $U(x, \varepsilon)$, there exists at least one point $z, z \neq x$ which is essential for $G$.

Proof Let for each $\varepsilon>0$ the open ball $U(x, \varepsilon)$ include at least one point $z, z \neq x$ essential for $G$ and put $\varepsilon_{z}=2^{-1} \rho(x, z)>0$. Then $U\left(z, \varepsilon_{z}\right) \subset U(x, \varepsilon)$ and therefore $\mu(U(x, \varepsilon) \cap G) \geq$ $\mu\left(U\left(z, \varepsilon_{z}\right) \cap G\right)>0$.

Remark 3.6 It is easy to see that if $G$ includes internal points, then all internal points of $G$ are essential for the set $G$. Every external point of $G$ is either nonessential for the set $G$, or an atom for the measure $\mu$. Moreover, if the set $G$ includes at least one essential for $G$ point, then we have $\mu(G)>0$.

Definition 3.7 ([11]) The sets $G, H \in B_{\Omega}$ will be called $\mu$-equivalent $G \sim^{\mu} H$ if $\mu(G \Delta$ $H)=0$.

Let $G, H \in B_{\Omega}$ be two arbitrary sets. If the set $G \Delta H$ includes at least one atom, then the sets $G$ and $H$ cannot be $\mu$-equivalent.

Definition 3.8 The set $G \in B_{\Omega}$ is called $\mu$-dense if each $x \in G$ is an essential for $G$ point.

Everywhere in our exposition below, we will assume that the measure $\mu$ is nonatomic.

Let $G$ be an arbitrary closed subset of $\Omega$. Denote by $G^{\mu}$ the set of all points $x \in G$, which are essential for $G$ and denote by $G^{v}$ the set $G^{v}=G \backslash G^{\mu}$.

Lemma 3.9 Let $G$ be an arbitrary closed subset of $\Omega$. Then the following statements are fulfilled:

1. The set $G^{\mu}$ is either empty or $\mu$-dense and a closed subset of $\Omega$.

2. The set $G^{v}$ is either empty or $G^{v} \subset \partial G$ and every point $x \in G^{v}$ is unessential for $G$. 
Proof 1 . If $G$ is closed and $\mu(G)=0$, then obviously $G^{\mu}=\varnothing$. Let $G^{\mu} \neq \varnothing$ and $x \in G$ be an arbitrary essential point. Then from the definition of the set $G^{\mu}$ it follows that $G^{\mu}$ is $\mu$-dense. Let $\left\{x_{n}\right\} \subset G^{\mu}$ be an arbitrary convergent sequence, $\lim _{n \rightarrow \infty} \rho\left(x_{n}, x_{0}\right)=0$ and $x_{0} \in G$. Then, for each $\varepsilon>0$ in the neighborhood $U\left(x_{0}, \varepsilon\right)=\left\{y \in \Omega \mid \rho\left(x_{0}, y\right)<\varepsilon\right\}$, there exists at least one essential point $x_{n}$ of $G$. Then Corollary 3.5 implies that $x_{0}$ is an essential point of $G$. Thus we can conclude that the set $G^{\mu}$ is a closed subset of $G$.

2. If $G^{\nu} \neq \varnothing$, then from point 1, Lemma 3.3 and Corollary 3.4 it follows that $G^{v} \subset \partial G$ and all points $x \in G^{v}$ are unessential for $G$.

Corollary 3.10 Let $G$ be an arbitrary closed subset of $\Omega$. Then if $G$ includes at least one essential point, we have that $\mu\left(G^{\mu}\right)>0$.

Proof Let $x_{0} \in G$ be an arbitrary essential point. Then, for each $\varepsilon>0$, we have $\mu(G \cap$ $\left.U\left(x_{0}, \varepsilon\right)\right)>0$ and therefore $\mu(G) \geq \mu\left(G \cap U\left(x_{0}, \varepsilon\right)\right)>0$.

Lemma 3.11 Let $G$ be a compact subset of $\Omega$, and $\mu(G)>0$. Then the set $G^{\mu}$ is a nonempty compact set, and the sets $G$ and $G^{\mu}$ are $\mu$-equivalent.

Proof Suppose that $G^{\mu}=\varnothing$. Then $G^{\nu}=G$, and according to Lemma 3.9, for $x \in G^{\nu}$, there exists $\varepsilon_{x}>0$ such that $\mu\left(U\left(x, \varepsilon_{x}\right) \cap G\right)=0$. Since $G$ is a compact subset of $\Omega$ and $\bigcup_{x \in G} U\left(x, \varepsilon_{x}\right)$ is an open cover of $G$, then there exist points $x_{1}, \ldots, x_{k} \in G$ and positive numbers $\varepsilon_{x_{1}}, \ldots, \varepsilon_{x_{k}}$ such that $\mu(G) \leq \mu\left(\bigcup_{i=1}^{k} U\left(x_{i}, \varepsilon_{i}\right) \cap G\right)=0$, which is impossible. Therefore $G^{\mu} \neq \varnothing$, and according to Lemma 3.9, the set $G^{\mu}$ is a closed subset of $G$, and we can conclude that $G^{\mu}$ is compact.

According to Lemma 3.9 and Lemma 3.3, for every $x \in G^{\nu}$, there exists $\varepsilon_{x}>0$ such that $\mu\left(U\left(x, \varepsilon_{x}\right) \cap G\right)=0$. Then $G^{v} \subseteq \bigcup_{x \in G^{\nu}} U\left(x, \varepsilon_{x}\right)$ and let $V_{1}, \ldots, V_{n}$ be open subsets of $\Omega$ such that $G^{\mu} \subseteq \bigcup_{i=1}^{n} V_{i}$. Therefore

$$
G \subseteq\left(\bigcup_{i=1}^{n} W_{i}\right) \cup\left(\bigcup_{x \in G^{v}} U\left(x, \varepsilon_{x}\right)\right)
$$

where $W_{i}=V_{i} \backslash\left(\bigcup_{x \in G^{\nu}} U\left(x, \varepsilon_{x}\right)\right)$ are open subsets of $\Omega$. Since $G$ is a compact set, then there exist points $x_{1}, \ldots, x_{k} \in G^{v}$ and positive numbers $\varepsilon_{x_{1}}, \ldots, \varepsilon_{x_{k}}$ such that $G^{v} \subseteq \bigcup_{i=1}^{k} U\left(x_{i}, \varepsilon_{x_{i}}\right)$, and for each $i$ we have $\mu\left(G^{v} \cap U\left(x_{i}, \varepsilon_{x_{i}}\right)\right)=0$. Therefore, $\mu\left(G^{v}\right)=0$ and the sets $G$ and $G^{\mu}$ are $\mu$-equivalent.

The next example illustrates the fact that the statement of Lemma 3.11 can be not true when the measure $\mu$ is atomic and the set $G$ is only closed, but not compact.

Example Let $x, y \in \Omega, x \neq y, x$ is not an atom and $y$ is an atom for the measure $\mu$. Define $r=2^{-1} \rho(x, y)$ and consider the closed set

$$
G=\{z \in \Omega \mid \rho(x, z) \leq r\} \cup\{y\}
$$

Obviously, $G^{\mu}=\{z \in \Omega \mid \rho(x, z) \leq r\}, \mu\left(G \Delta G^{\mu}\right)=\mu(\{y\})>0$, and therefore the sets $G^{\mu}$ and $G$ are not $\mu$-equivalent. 
Lemma 3.12 Let, for the map $M: \Omega \rightarrow 2^{\Omega}$, conditions (A1) and (A2) hold. Then, for every $x \in \Omega$ for which $\mu\left(M_{x}\right)>0$, there exists $\delta>0$ such that for each $s \in \Omega$ with $\rho(x, s)<\delta$, we have $M_{x} \cap M_{s} \neq \varnothing$.

Proof Suppose that the statement of Lemma 3.12 is not true. Then there exist a strictly decreasing sequence from positive numbers $\left\{\delta_{n}\right\}_{n=1}^{\infty}$ with $\lim _{n \rightarrow \infty} \delta_{n}=0$ and a sequence $\left\{y_{n}\right\}_{n=1}^{\infty} \subset \Omega, y_{n} \in U\left(x, \delta_{n}\right)$ with $M_{x} \cap M_{y_{n}}=\varnothing$ for each $n \geq 1$. Since $\lim _{n \rightarrow \infty} \rho\left(x, y_{n}\right)=0$, then from condition (A2) it follows that $\lim _{n \rightarrow \infty} \mu\left(M_{x} \Delta M_{y_{n}}\right)=0$. On the other hand, since $M_{x} \cap M_{y_{n}}=\varnothing$ for each $n \geq 1$, then we have that $\mu\left(M_{x} \Delta M_{y_{n}}\right) \geq \mu\left(M_{x}\right)>0$, which is impossible.

Corollary 3.13 Let the conditions of Lemma 3.12 hold. Then, for each $x \in \Omega$, there exists $\delta>0$ such that for each $s \in \Omega$ with $\rho(x, s)<\delta$, we have $\mu\left(M_{s}\right)>0$.

Proof Since $\mu\left(M_{x}\right)>0$, then condition (A2) implies that there exists $\delta>0$ such that for each $s \in \Omega$ with $\rho(x, s)<\delta$, we have

$$
\mu\left(M_{x} \backslash M_{s}\right) \leq \mu\left(M_{x} \Delta M_{s}\right)<\frac{\mu\left(M_{x}\right)}{4} .
$$

Then from $\mu\left(M_{x}\right)=\mu\left(M_{x} \cap M_{s}\right)+\mu\left(M_{x} \backslash M_{s}\right)$ it follows that

$$
\mu\left(M_{x} \cap M_{s}\right) \geq \frac{3 \mu\left(M_{x}\right)}{4}>0 .
$$

For every map $M: \Omega \rightarrow 2^{\Omega}$, we define the associated map $M^{\mu}: \Omega \rightarrow 2^{\Omega}$ for each $x \in \Omega$ by the following relation $M_{x}^{\mu}=\left(M_{x}\right)^{\mu}$.

Lemma 3.14 ([11]) Let, for the map $M: \Omega \rightarrow 2^{\Omega}$, conditions (A) hold. Then, for the map $M^{\mu}$, conditions (A) hold too.

Remark 3.15 From Lemma 3.14 and Lemma 3.11 it follows that for every map $M: \Omega \rightarrow 2^{\Omega}$ for which conditions (A) hold, without loss of generality, we can use for every $x \in \Omega$ as a domain of integration instead of the set $M_{x}$ its $\mu$-equivalent set $M_{x}^{\mu}$ which is $\mu$-dense.

Let the map $M: \Omega \rightarrow 2^{\Omega}$ be arbitrary and denote by $\operatorname{Ker} M$ the set $\operatorname{Ker} M=\{x \in \Omega \mid$ $\left.\mu\left(M_{x}\right)=0\right\}$.

Lemma 3.16 Let, for the map $M: \Omega \rightarrow 2^{\Omega}$, the following conditions be fulfilled:

1. Conditions (A) hold.

2. $\Omega$ is a connected metric space and $0<\mu(\Omega) \leq \infty$.

Then, for every $x \in \Omega$, the following statements are true:

(i) $\mu\left(M_{x}\right)<\infty$;

(ii) $\operatorname{Ker} M \cap M_{x} \neq \varnothing$.

Proof (i) Let us consider the set $\Omega_{1}=\left\{x \in \Omega \mid \mu\left(M_{x}\right)<\infty\right\}$ and define $\Omega_{2}=\Omega \backslash \Omega_{1}$. Condition (A4) implies that $\Omega_{1} \neq \varnothing$, and let us assume that $\Omega_{2} \neq \varnothing$ too.

First we will prove that $\Omega_{1}$ is a closed subset of $\Omega$. Let $y \in \partial \Omega_{1}$ be an arbitrary point. Then there exists a sequence $\left\{x_{n}\right\}_{n=1}^{\infty} \subset$ int $\Omega_{1}$ such that $\lim _{n \rightarrow \infty} \rho\left(x_{n}, y\right)=0$. Since 
$\lim _{n, m \rightarrow \infty} \mu\left(M_{x_{n}} \Delta M_{x_{m}}\right)=0$, then there exist a number $n_{0}$ and a constant $C>0$ such that $\mu\left(M_{x_{n}}\right) \leq C$ for each $n \geq n_{0}$. For an arbitrary number $\varepsilon>0$ from condition (A2) it follows that there exists a number $n_{1}(\varepsilon) \geq n_{0}$ such that for each $n \geq n_{1}(\varepsilon)$ we have $\mu\left(M_{x_{n}} \Delta M_{y}\right)<\varepsilon$. Then $\mu\left(M_{y}\right) \leq \mu\left(M_{x_{n}}\right)+\mu\left(M_{x_{n}} \Delta M_{y}\right) \leq C+\varepsilon<\infty$ and therefore $y \in \Omega_{1}$. Thus we proved that $\Omega_{1}$ is a closed subset of $\Omega$. It is easy to see that for each $z \in \partial \Omega_{2}$ from condition (A2) it follows that $\mu\left(M_{z}\right)=+\infty$. Then $\Omega_{2}$ is also a closed set and therefore $\Omega=\Omega_{1} \cup \Omega_{2}$, where $\Omega_{1} \cap \Omega_{2}=\varnothing$, which is impossible. Thus we can conclude that $\Omega_{2}=\varnothing$.

(ii) Assume that there exists some $y \in \Omega$ such that $M_{y} \cap \operatorname{Ker} M=\varnothing$. From condition (A2) and (i) it follows that the function $\mu \circ M: \Omega \rightarrow[0, \infty)$ is continuous in $\Omega$. Since $M_{y}$ is compact, there exists $z \in M_{y}$ such that

$$
\mu\left(M_{z}\right)=\min _{s \in M_{y}} \mu\left(M_{s}\right) \quad \text { and } \quad \mu\left(M_{z}\right)>0 .
$$

Let $x \in \Omega$ be an arbitrary point for which $M_{x} \cap M_{z} \neq \varnothing$. Then either

$$
\mu\left(M_{x} \cap M_{z}\right)=\mu\left(M_{z}\right) \quad \text { or } \quad M_{z} \backslash M_{x} \neq \varnothing \quad \text { and } \quad \mu\left(M_{z} \backslash M_{x}\right)>0 .
$$

In the second case we have that $0 \leq \mu\left(M_{x} \cap M_{z}\right)<\mu\left(M_{z}\right)$ and from condition (A3) it follows that there exists a point $y_{*} \in M_{x} \cap M_{z}$ such that

$$
M_{y_{*}} \subset M_{x} \cap M_{z} \subset M_{y}
$$

and the inequality $\mu\left(M_{y_{*}}\right)<\mu\left(M_{z}\right)$ holds, which is impossible. Then, for every point $x \in \Omega$, we have that either $M_{x} \cap M_{z}=\varnothing$ or $\mu\left(M_{x} \cap M_{z}\right)=\mu\left(M_{z}\right)$.

Define $G=\left\{x \in \Omega \mid \mu\left(M_{x} \cap M_{z}\right)=\mu\left(M_{z}\right)\right\}$ and $H=\Omega \backslash G$. We will prove that $G$ is closed. Since $G \neq \varnothing$, let $\left\{x_{n}\right\}_{n=1}^{\infty} \subseteq G$ be an arbitrary convergent sequence with boundary $s \in \Omega$, i.e., $\lim _{n \rightarrow \infty} \rho\left(x_{n}, s\right)=0$. Then we have $\lim _{n \rightarrow \infty} \mu\left(M_{x_{n}} \Delta M_{s}\right)=0$, and there exists $n_{0}$ integer such that for each $n \geq n_{0}$ the inequality $\mu\left(M_{x_{n}} \Delta M_{s}\right)<\frac{\mu\left(M_{z}\right)}{2}$ holds.

If we assume that $0 \leq \mu\left(M_{s} \cap M_{z}\right)<\mu\left(M_{z}\right)$, then $M_{s} \cap M_{z}=\varnothing$, and therefore we have that the following estimation

$$
\mu\left(M_{x_{n}} \Delta M_{s}\right) \geq \mu\left(M_{x_{n}} \backslash M_{s}\right) \geq \mu\left(M_{z} \backslash M_{s}\right) \geq \mu\left(M_{z}\right)
$$

holds, which is impossible. Therefore $\mu\left(M_{s} \cap M_{z}\right)=\mu\left(M_{z}\right)$, i.e., $s \in G$.

Since $H \neq \varnothing$ and $\Omega$ is connected, then $H$ cannot be a closed set. There exist a point $s \in \partial G$ and a sequence $\left\{t_{n}\right\}_{n=1}^{\infty} \subseteq H$ such that $\lim _{n \rightarrow \infty} \rho\left(t_{n}, s\right)=0$, and therefore $\lim _{n \rightarrow \infty} \mu\left(M_{t_{n}} \Delta M_{s}\right)=0$. There exists $n_{0}$ integer such that for each $n \geq n_{0}$ we have $\mu\left(M_{t_{n}} \Delta M_{s}\right)<\frac{\mu\left(M_{z}\right)}{2}$. Since the sequence $\left\{t_{n}\right\}_{n=1}^{\infty} \subseteq H$, then we have that $0 \leq \mu\left(M_{t_{n}} \cap M_{z}\right)<$ $\mu\left(M_{z}\right)$ and therefore $M_{t_{n}} \cap M_{z}=\varnothing$. Then the following estimation $\mu\left(M_{t_{n}} \Delta M_{s}\right) \geq \mu\left(M_{s} \backslash\right.$ $\left.M_{t_{n}}\right) \geq \mu\left(M_{z}\right)$ holds, which is impossible.

Remark 3.17 The assertion of Lemma 3.16 was proved in [11] with the additional assumption that all sets $M_{x}$ are $\mu$-dense.

We will say that condition (AM) is fulfilled if the following condition holds:

(AM) For each $x \in \Omega$, the set $M_{x}$ is $\mu$-dense. 
Before considering the next important theorem, we draw attention to that from the viewpoint of the applications the case when $\operatorname{Ker} M=\Omega$ is meaningless.

Theorem 3.18 Let, for the map $M: \Omega \rightarrow 2^{\Omega}$, the following conditions be fulfilled:

1. Conditions (A) and (AM) hold.

2. $\Omega$ is a connected metric space and $0<\mu(\Omega) \leq \infty$.

Then, for every $x \in \Omega$ for which $M_{x} \backslash \operatorname{Ker} M \neq \varnothing$, there exist a point $y_{0} \in \partial \operatorname{Ker} M \cap M_{x}$ and a sequence $\left\{y_{n}\right\}_{n=1}^{\infty} \subseteq M_{x} \backslash \operatorname{Ker} M$ such that $\lim _{n \rightarrow \infty} \rho\left(y_{n}, y_{0}\right)=0$.

Proof Let $x \in \Omega$ be an arbitrary point, for which $M_{x} \backslash \operatorname{Ker} M \neq \varnothing$. In virtue of Lemma 3.16, there exists at least one connected component $M^{\text {co }}$ of $M_{x}, M^{\text {co }} \subseteq M_{x}$ such that $M^{\text {co }} \backslash$ $\operatorname{Ker} M \neq \varnothing$. For the closure of $M^{\mathrm{co}}$, we have that $\mathrm{cl}_{\Omega}\left(M^{\mathrm{co}}\right) \subseteq M_{x}$, then $\mathrm{cl}_{\Omega}\left(M^{\mathrm{co}}\right)$ is compact. Since $\operatorname{cl}_{\Omega}\left(M^{\mathrm{co}}\right)$ is compact, then if we assume that $\operatorname{cl}_{\Omega}\left(M^{\mathrm{co}}\right) \cap \operatorname{Ker} M=\varnothing$, then there exists $z \in \operatorname{cl}_{\Omega}\left(M^{\mathrm{co}}\right)$ such that $\mu\left(M_{z}\right)=\min _{y \in \mathrm{cl}_{\Omega}\left(M^{\mathrm{co}}\right)} \mu\left(M_{y}\right)$ and $\mu\left(M_{z}\right)>0$, which is impossible.

Define $G=\left\{y \in \Omega \mid \mu\left(M_{y} \cap M_{z}\right)=\mu\left(M_{z}\right)\right\}$ and $H=\Omega \backslash G$. In a similar way as in the proof of Lemma 3.16, we can prove that $G$ is closed, $G \neq \varnothing$ and $H \neq \varnothing$ too. Moreover, for each $y \in H$, we have $\mu\left(M_{y} \cap M_{z}\right)=0$. Then there exist a point $s \in \partial G$ and a sequence $\left\{t_{n}\right\}_{n=1}^{\infty} \subseteq H$ such that $\lim _{n \rightarrow \infty} \rho\left(t_{n}, s\right)=0$ and therefore $\lim _{n \rightarrow \infty} \mu\left(M_{t_{n}} \Delta M_{s}\right)=0$. In the same way as in the proof of Lemma 3.16, we conclude that this is impossible.

Then we can conclude that $\operatorname{cl}_{\Omega}\left(M^{\mathrm{co}}\right) \cap \operatorname{Ker} M \neq \varnothing$, and therefore there exists at least a point $y_{0} \in \partial \operatorname{Ker} M \cap M_{x}$. Then, since $\operatorname{cl}_{\Omega}\left(M^{\text {co }}\right)$ is connected, there exists $\left\{y_{n}\right\}_{n=1}^{\infty} \subset$ $\operatorname{cl}_{\Omega}\left(M^{\mathrm{co}}\right) \backslash \operatorname{Ker} M$ such that $\lim _{n \rightarrow \infty} \rho\left(y_{n}, y_{0}\right)=0$.

Theorem 3.19 Let conditions (A) and (AM) hold. Then, for every point $x \in \Omega$ and each $\varepsilon>0$, there exists $\delta(x, \varepsilon)>0$ such that for $s \in U(x, \delta)$ we have that $M_{x} \subset U\left(M_{s}, \varepsilon\right)$ and $M_{s} \subset$ $U\left(M_{x}, \varepsilon\right)$.

Proof Let $\varepsilon>0, x \in \Omega$ be an arbitrary point and suppose that $M_{x} \neq \varnothing$ (the case $M_{x}=\varnothing$ is trivial). If we assume that $M_{x} \backslash U\left(M_{s}, \varepsilon\right) \neq \varnothing$, then there exists a decreasing sequence of positive numbers $\left\{\delta_{n}\right\}_{n=1}^{\infty}, \lim _{n \rightarrow \infty} \delta_{n}=0$ such that for each $\delta_{n}$, there exist points $s_{n} \in U\left(x, \delta_{n}\right)$ and $x_{n} \in M_{x} \backslash U\left(M_{s_{n}}, \varepsilon\right)$ such that $\rho\left(x_{n}, M_{s_{n}}\right)>\varepsilon$ for each $n \geq 1$. This implies that for each $n \geq 1$ we have $U\left(x_{n}, \varepsilon\right) \cap M_{z_{n}}=\varnothing$ and therefore the inequality

$$
\mu\left(M_{x} \backslash M_{s_{n}}\right) \geq \mu\left(M_{x} \cap U\left(x_{n}, \varepsilon\right)\right)
$$

holds. Taking into account that $M_{x}$ is compact, there exists a convergent subsequence $\left\{x_{n_{k}}\right\}_{k=1}^{\infty}$ such that $\lim _{k \rightarrow \infty} \rho\left(x_{n_{k}}, x_{0}\right)=0$, where $x_{0} \in M_{x}$. Since $\lim _{k \rightarrow \infty} \rho\left(s_{n_{k}}, x_{0}\right)=0$, then from the last inequality above and condition (A2) the relation $\mu\left(U\left(x_{0}, \varepsilon\right) \cap M_{x}\right)=0$ follows. Therefore $x_{0}$ is an unessential point of $M_{x}$, which is impossible. Then there exists $\delta(x, \varepsilon)>0$ such that for every $s \in U(x, \delta)$ the inclusion $M_{x} \subset U\left(M_{s}, \varepsilon\right)$ holds.

Let $s \in U(x, \delta)$ be an arbitrary fixed point. Then we have $\rho\left(M_{s}, M_{s}\right)<\varepsilon$ and therefore $M_{s} \subset U\left(M_{x}, \varepsilon\right)$.

Remark 3.20 For the sets $M_{x}$ which are $\mu$-dense, Theorem 3.19 illustrates the important fact that if these sets are close in the measure sense, they are close in the metric sense too.

Definition 3.21 We say that the set $G \subseteq \Omega$ is $M$-star if for every $x \in G$ the inclusion $M_{x} \subseteq G$ holds. 
Remark 3.22 It is easy to see that condition (A3) implies that for each $x \in \Omega$ the set $M_{x}$ is an $M$-star set. Moreover, the union and the intersection of an arbitrary family of $M$-star sets are the $M$-star set. The set $\operatorname{Ker} M$ is an $M$-star set too.

\section{Main results}

Let the map $M: \Omega \rightarrow 2^{\Omega}$ satisfy conditions (A) and (AM). For each $M$-star set $\Omega_{*} \subset \Omega$, we denote by $B C\left(\Omega_{*}\right)$ the normed space of all functions $f: \Omega_{*} \rightarrow B$, for which

$$
\|f\|_{\Omega_{*}}=\sup _{x \in \Omega_{*}}\|f(x)\|_{B}<\infty \quad \text { and } \quad f \in C\left(M\left(\Omega_{*}\right), B\right)
$$

where $M\left(\Omega_{*}\right)=\operatorname{cl}_{\Omega_{*}}\left(\bigcup_{x \in \Omega_{*}} M_{x}\right)$.

In our discussion below we will assume that for the operator

$$
Q: \Omega \times \Omega \times B \rightarrow B,
$$

some of the following conditions are fulfilled.

(S1) For every $M$-star compact set $\Omega_{*} \subset \Omega$, the operator $Q$ is continuous in the set $\Omega_{*} \times \Omega_{*} \times B$.

(S2) For each $x \in \Omega$ and every $f \in C\left(M_{x}, B\right)$, there exist numbers $\delta\left(M_{x}, f\right)>0$ and $L\left(M_{x}, f, \delta\right)>0$ such that for every functions $g \in C\left(M_{x}, B\right)$ for which $\|f-g\|_{M_{x}}<\delta$, the following inequality holds:

$$
\sup _{(s, y) \in M_{x} \times M_{x}}\|Q(s, y, f(y))-Q(s, y, g(y))\|_{B} \leq L\left(M_{x}, f, \delta\right)\|f-g\|_{M_{x}} .
$$

(S3) For every $x \in \Omega$ and arbitrary $r>0$, there exists a constant $L\left(M_{x}, r\right)>0$ such that for every two functions $f, g \in \bar{U}_{x}(r)=\left\{f \in C\left(M_{x}, B\right) \mid\|f\|_{M_{x}} \leq r\right\}$, the following inequality holds:

$$
\sup _{(s, y) \in M_{x} \times M_{x}}\|Q(s, y, f(y))-Q(s, y, g(y))\|_{B} \leq L\left(M_{x}, r\right)\|f-g\|_{M_{x}} .
$$

(S4) For each $M$-star continuum $\Omega_{*} \subset \Omega$, there exists a number $L\left(\Omega_{*}\right)>0$ such that for every two functions $f_{1}, f_{2} \in C\left(\Omega_{*}, B\right)$ and for each $x, y \in \Omega_{*}$, the following inequality holds:

$$
\left\|Q\left(x, y, f_{1}(y)\right)-Q\left(x, y, f_{2}(y)\right)\right\|_{B} \leq L\left(\Omega^{*}\right)\left\|f_{1}(y)-f_{2}(y)\right\|_{B} .
$$

Consider the operator $K$ defined by the equation

$$
K f(x)=p(x)+\int_{M_{x}} Q(x, y, f(y)) d \mu_{y},
$$

where $f, p \in C(\Omega, B), x \in \Omega$. If for the operator $Q$ condition (S1) holds, then the integral in (4.1) exists; see, e.g., [17, 18].

Lemma 4.1 Let $x \in \Omega$ be an arbitrary point and the following conditions be fulfilled:

1. Conditions (A), (AM), (S1) and (S2) hold. 
2. $\Omega$ is a connected metric space and $0<\mu(\Omega) \leq \infty$.

Then the operator $K$ defined by equality (4.1) maps $C\left(M_{x}, B\right) \rightarrow C\left(M_{x}, B\right)$ continuously for each $p \in C\left(M_{x}, B\right)$.

Proof Let $s_{0} \in M_{x}$ be an arbitrary fixed point, and let $\left\{s_{n}\right\}_{n=1}^{\infty} \subset M_{x}$ be an arbitrary sequence such that $\lim _{n \rightarrow+\infty} \rho\left(s_{n}, s_{0}\right)=0$. If $f \in C\left(M_{x}, B\right)$ is an arbitrary fixed element, then

$$
\begin{aligned}
\left\|K f\left(s_{n}\right)-K f\left(s_{0}\right)\right\|_{B} \leq & \left\|p\left(s_{n}\right)-p\left(s_{0}\right)\right\|_{B} \\
& +\int_{M_{s_{n}} \cap M_{s_{0}}}\left\|Q\left(s_{n}, y, f(y)\right)-Q\left(s_{0}, y, f(y)\right)\right\|_{B} d \mu_{y} \\
& +\int_{M_{s_{n} \backslash M_{s_{0}}}\left\|Q\left(s_{n}, y, f(y)\right)\right\|_{B} d \mu_{y}} \\
& +\int_{M_{s_{0}} \backslash M_{s_{n}}}\left\|Q\left(s_{0}, y, f(y)\right)\right\|_{B} d \mu_{y} .
\end{aligned}
$$

Let $\varepsilon>0$ be an arbitrary number. Define $B(f)=M_{x} \times M_{x} \times f\left(M_{x}\right)$ and $T=\sup _{(s, y, v) \in B(f)} \| Q(s$, $y, v) \|_{B}$. From conditions (A1) and (S1) it follows that a number $n_{0}$ exists such that for each $n \geq n_{0}$ and $y \in M_{s_{0}}$, the inequalities

$$
\begin{aligned}
& \sup _{n \geq n_{0}, y \in M_{s_{0}}}\left\|Q\left(s_{n}, y, f(y)\right)-Q\left(s_{0}, y, f(y)\right)\right\|_{B}<\frac{\varepsilon}{4 \mu\left(M_{s_{0}}\right)}, \\
& \left\|p\left(s_{n}\right)-p\left(s_{0}\right)\right\|_{B}<\frac{\varepsilon}{4}
\end{aligned}
$$

and

$$
\mu\left(M_{s_{n}} \Delta M_{s_{0}}\right) \leq \frac{\varepsilon}{4 T}
$$

hold. Therefore, from (4.2) it follows that for each $n \geq n_{0}$, we have

$$
\left\|(K f)\left(s_{n}\right)-(K f)\left(s_{0}\right)\right\|_{B}<\varepsilon .
$$

Let $\left\{f_{n}\right\}_{n=1}^{\infty} \subset C\left(M_{x}, B\right)$ be an arbitrary sequence such that

$$
\lim _{n \rightarrow \infty}\left\|f_{n}-f\right\|_{M_{x}}=0
$$

The function $\mu \circ M: \Omega \rightarrow[0, \infty)$ is continuous in $\Omega$ and then there exists a point $x_{*} \in M_{x}$ such that $\mu\left(M_{x_{*}}\right)=\sup _{s \in M_{x}} \mu\left(M_{s}\right)$. Moreover, from condition (S2) it follows that there exists a number $n_{0}=n_{0}(\varepsilon)$ such that for each $n \geq n_{0}$, we have $\left\|f_{n}-f\right\|_{M_{x}}<\delta$ and the estimation

$$
\sup _{(s, y) \in M_{x} \times M_{x}}\left\|Q\left(s, y, f_{n}(y)\right)-Q(s, y, f(y))\right\|_{B} \leq L\left(M_{x}, f, \delta\right)\left\|f_{n}-f\right\|_{M_{x}}
$$

holds. Since $\lim _{n \rightarrow \infty}\left\|f_{n}-f\right\|_{M_{x}}=0$, then for each $\varepsilon>0$, there exists a number $n_{1}=n_{1}(\varepsilon) \geq$ $n_{0}$ such that for each $n \geq n_{1}$, we have

$$
\left\|f_{n}-f\right\|_{M_{x}}<\min \left(\delta, \varepsilon\left(\mu\left(M_{x_{*}}\right) L\left(M_{x}, f, \delta\right)\right)^{-1}\right) .
$$


Therefore, from (4.3) it follows that

$$
\begin{aligned}
\left\|K f_{n}-K f\right\|_{M_{x}} & \leq \sup _{s \in M_{x}} \int_{M_{s}}\left\|Q\left(s, y, f_{n}(y)\right)-Q(s, y, f(y))\right\|_{B} d \mu_{y} \\
& \leq \mu\left(M_{x_{*}}\right) \sup _{s \in M_{x}}\left(\sup _{y \in M_{x}}\left\|Q(s, y, f(y))-Q\left(s, y, f_{n}(y)\right)\right\|_{B}\right) \\
& \leq \mu\left(M_{x_{*}}\right) L\left(M_{x}, f, \delta\right)\left\|f_{n}-f\right\|_{M_{x}}<\varepsilon .
\end{aligned}
$$

Let $x \in \Omega$ be an arbitrary point. Then, for every $f \in C\left(M_{x}, B\right)$, there exist two points $s_{m}^{x}, s_{M}^{x} \in M_{x}$ such that

$$
\left\|f\left(s_{m}^{x}\right)\right\|_{B}=\min _{s \in M_{x}}\|f(s)\|_{B} \quad \text { and } \quad\left\|f\left(s_{M}^{x}\right)\right\|_{B}=\max _{s \in M_{x}}\|f(s)\|_{B} .
$$

Definition 4.2 A continuous function $f: \Omega \rightarrow B$ is called an extension of the function $f \in C\left(M_{x}, B\right)$ if $F(s)=f(s)$ for each $s \in M_{x}$. If in addition for some extension $F(s)$ and each $s \in \Omega$ the following inequalities

$$
\left\|f\left(s_{m}\right)\right\|_{B} \leq\|F(s)\|_{B} \leq\left\|f\left(s_{M}\right)\right\|_{B}
$$

hold, then such an extension will be called a middle extension.

Lemma 4.3 Let conditions (A) and (AM) be fulfilled. Then, for each $x \in \Omega$, every function $f \in C\left(M_{x}, B\right)$ has at least one middle extension.

Proof Let $x \in \Omega$ and $f \in C\left(M_{x}, B\right)$ be an arbitrary function. Denote by $F \in C(\Omega, B)$ the extension of the function $f-f\left(s_{m}\right)$ which exists according to the Dugundji extension theorem ([19], Theorem 4.1). Define $F_{*} \in C(\Omega, B)$ by $F_{*}(s)=F(s)$ for $s \in M_{x}$ such that

$$
0 \leq\|F(s)\|_{B} \leq\left\|f\left(s_{M}\right)\right\|_{B}-\left\|f\left(s_{m}\right)\right\|_{B}
$$

and $F_{*}(s)=f\left(s_{M}\right)-f\left(s_{m}\right)$ for $s \in M_{x}$ such that

$$
\|F(s)\|_{B}>\left\|f\left(s_{M}\right)\right\|_{B}-\left\|f\left(s_{m}\right)\right\|_{B} .
$$

Since the function

$$
\bar{F}_{*}=F_{*}+f\left(s_{m}\right)
$$

is an extension of $f$ for which the inequalities

$$
\left\|f\left(s_{m}\right)\right\|_{B} \leq\|\bar{F}(s)\|_{B} \leq\left\|f\left(s_{M}\right)\right\|_{B}
$$

hold for each $s \in \Omega$, then $\bar{F}_{*}$ is a middle extension of the function $f \in C\left(M_{x}, B\right)$.

Definition 4.4 We say that equation (2.1) has a local solution in some $M$-star set $\Omega_{*} \subset \Omega$ for some $p \in B C\left(\Omega_{*}, B\right)$ if there exist a point $x_{p} \in \Omega_{*}$ and a function $f \in C\left(M_{x_{p}}, B\right)$ for which $\mu\left(M_{x_{p}}\right)>0$ and $f$ satisfies equation (2.1) for each $s \in M_{x_{p}}$. If $p, f \in C\left(\Omega_{*}, B\right)$ and $f$ satisfies equation (2.1) for each $x \in \Omega_{*}$, then we say that $f$ is a solution of (2.1) in $\Omega_{*} \subset \Omega$. 
Theorem 4.5 Let the following conditions be fulfilled:

1. Conditions (A), (AM), (S1) and (S3) hold.

2. $\Omega$ is a connected metric space and $0<\mu(\Omega) \leq \infty$.

3. $\operatorname{Ker} M \subset \Omega$ (the case when $\operatorname{Ker} M=\Omega$ is trivial).

Then, for every $x \in \Omega$ such that $M_{x} \backslash \operatorname{Ker} M \neq \varnothing$ and for every $p \in C\left(M_{x_{p}}, B\right)$, equation (2.1) has at least one local solution in $M_{x}$.

Proof From condition 3, Lemma 3.16 and Theorem 3.18 it follows that there exists $x \in \Omega$ such that $\operatorname{Ker} M \cap M_{x} \neq \varnothing$ and $M_{x} \backslash \operatorname{Ker} M \neq \varnothing$. Moreover, there exist a point $y_{0} \in \partial \operatorname{Ker} M \cap$ $M_{x}$ and a sequence $\left\{y_{n}\right\}_{n=1}^{\infty} \subseteq M_{x} \backslash \operatorname{Ker} M$ such that $\lim _{n \rightarrow \infty} \rho\left(y_{n}, y_{0}\right)=0$. Denote by $U_{n}(r)$ for every $n \geq 1$ the set

$$
U_{n}(r)=\left\{f \mid f \in C\left(M_{y_{n}}, B\right),\|f\|_{M_{y_{n}}} \leq r\right\} .
$$

According to Lemma 4.3, for every function $f \in C\left(M_{y_{n}}, B\right)$ and for each $n \geq 1$, there exists a middle extension $F_{y_{n}} \in C\left(M_{x}, B\right)$ such that $\left\|F_{y_{n}}\right\|_{M_{x}}=\|f\|_{M_{y_{n}}}$. Therefore, the set $\bar{U}_{n}(r)=$ $\left\{F_{y_{n}} \mid f \in U_{n}(r)\right\} \subset \bar{U}_{x}(r)$ for every $n \geq 1$.

Let us define

$$
\begin{aligned}
& P=\max _{s \in M_{x}}\|p(s)\|_{B^{\prime}} \\
& Q_{0}=\sup _{(s, y) \in M_{x} \times M_{x}}\|Q(s, y, 0)\|_{B}
\end{aligned}
$$

and $r>P$ is an arbitrary number and define for every $n \geq 1$ the operator $K_{n}$ by the following equality:

$$
K_{n} f(s)=p(s)+\int_{M_{s}} Q(s, y, f(y)) d \mu_{y}, \quad s \in M_{y_{n}},
$$

where $f \in C\left(M_{y_{n}}, B\right), p \in C\left(M_{x}, B\right)$.

From Lemma 4.1 it follows that for each $n \geq 1$ the operator $K_{n}$ maps $C\left(M_{y_{n}}, B\right)$ into $C\left(M_{y_{n}}, B\right)$ continuously, and from (4.4) and (S3) it follows that the inequality

$$
\begin{aligned}
\left\|K_{n} f\right\|_{M_{y_{n}}} & \leq P+\sup _{s \in M_{y_{n}}} \int\|Q(s, y, f(y))-Q(s, y, 0)+Q(s, y, 0)\| d \mu_{y} \\
& \leq P+\mu\left(M_{y_{n}}\right)\left(L\left(M_{x}, r\right)\|f\|_{M_{y_{n}}}+Q_{0}\right) \\
& \leq P+\mu\left(M_{y_{n}}\right)\left(L\left(M_{x}, r\right) r+Q_{0}\right)
\end{aligned}
$$

holds for each $f \in U_{n}(r)$ and every $n \geq 1$. Since $\lim _{n \rightarrow \infty} \rho\left(y_{n}, y_{0}\right)=0$, then there exists a number $n_{0}=n_{0}\left(y_{0}, r\right)$ such that for every $n \geq n_{0}$ from (4.5) it follows that the inequalities

$$
\begin{aligned}
& P+\mu\left(M_{y_{n}}\right)\left(L\left(M_{x}, r\right) r+Q_{0}\right)<r, \\
& \mu\left(M_{y_{n}}\right) L\left(M_{x}, r\right) \leq q<1
\end{aligned}
$$

hold. Obviously, first of them implies that $K_{n}\left(U_{n}(r)\right) \subseteq U_{n}(r)$. 
Define $a=y_{n_{1}}$ for some fixed $n_{1} \geq n_{0}$, and let $f, g \in U_{n_{1}}(r)$ be arbitrary functions. If we denote by $F_{a}$ and $G_{a}$ their middle extensions on $M_{x}$, then from (4.6) it follows the estimation

$$
\begin{aligned}
\left\|K_{a} f-K_{a} g\right\|_{M_{a}} & \leq \mu\left(M_{a}\right) \sup _{(s, y) \in M_{a} \times M_{a}}\|Q(s, y, f(y))-Q(s, y, g(y))\|_{B} \\
& \leq \mu\left(M_{a}\right) \sup _{(s, y) \in M_{a} \times M_{a}}\left\|Q\left(s, y, F_{a}(y)\right)-Q\left(s, y, G_{a}(y)\right)\right\|_{B} \\
& \leq \mu\left(M_{a}\right) L\left(M_{x}, r\right)\left\|F_{a}-G_{a}\right\|_{M_{x}}=\mu\left(M_{a}\right) L\left(M_{x}, r\right)\|f-g\|_{M_{a}} \\
& \leq q\|f-g\|_{M_{a}} .
\end{aligned}
$$

Therefore, the operator $K_{a}$ maps $U_{n_{1}}(r)$ into $U_{n_{1}}(r)$ and is a contraction. Therefore, equation (2.1) has a solution $f \in C\left(M_{a}, B\right)$. Moreover, since $y_{n_{1}} \in M_{x} \backslash \operatorname{Ker} M$, then $\mu\left(M_{a}\right)>0$.

Remark 4.6 Generally speaking, if the set $M_{a}$ is not connected, then the local solution of equation (2.1) can be not unique. The next example confirms that the connectivity of the set $M_{a}$ is essential for the uniqueness of the local solution of equation (2.1).

Example Let $\Omega=[0, \infty), B=\mathbb{R}, M_{x}=[0, x] \cup[1,1+x]$ for $x \in[0,1], M_{x} \in[0,1+x]$ for $x \in$ $[1, \infty)$ and $p \equiv 1$ for $x \in[0, \infty)$. Then, for each $x \in(0,1)$ according to Theorem 4.5 , there exists $a_{x} \in(0, x)$ such that equation (2.1) has a solution $f \in C\left(M_{a_{x}}, \mathbb{R}\right)$ in $M_{a_{x}}$. Moreover, since $p \equiv 1$ for $x \in[0, \infty)$, then from conditions (A) it follows that if we choose $a_{x} \in(0, x)$ small enough, then $f(s)>0$ for $s \in\left(0, a_{x}\right)$. By the Urison lemma, there exists a continuous function $h: \Omega \rightarrow[0,1]$ such that $h(s) \equiv 0$ for $s \in\left[1,1+a_{x}\right]$ and $h(s) \equiv 1$ when $s \in\left[0, a_{x}\right]$. Then, obviously, the function $w(s)=h(s) f(s)$ is another solution of equation (2.1) in $M_{a_{x}}$.

Theorem 4.7 Let the set $\Omega^{*} \subset \Omega$ be an arbitrary M-star continuum, $\mu\left(\Omega^{*}\right)<+\infty$ and the following conditions be fulfilled:

1. The metric space $\Omega$ is connected and $0<\mu(\Omega) \leq \infty$.

2. Conditions (A), (S1), (S4) and (AM) hold and for each $x \in \Omega^{*}$ the sets $M_{x}$ are connected.

Then, for each $p \in C\left(\Omega^{*}, B\right)$, equation (2.1) has exactly one solution $f \in C\left(\Omega^{*}, B\right)$.

Proof Suppose that $f_{1}, f_{2} \in C\left(\Omega^{*}, B\right)$ are two different solutions of equation (2.1). Then from (2.1) it follows that for each $x \in \Omega_{*}$ the inequality

$$
\left\|f_{1}(x)-f_{2}(x)\right\|_{B} \leq \int_{M_{x}}\left\|Q\left(x, y, f_{1}(y)\right)-Q\left(x, y, f_{2}(y)\right)\right\|_{B} d \mu_{y}
$$

holds. From the last equation and condition (S4) it follows that for each $x \in \Omega_{*}$ the inequality

$$
\left\|f_{1}(x)-f_{2}(x)\right\|_{B} \leq L\left(\Omega_{*}\right) \int_{M_{x}}\left\|f_{1}(y)-f_{2}(y)\right\|_{B} d \mu_{y}
$$

holds. Using Theorem 2 and Theorem 3 from [8] we get $\left\|f_{1}(x)-f_{2}(x)\right\|_{B}=0$ for each $x \in \Omega_{*}$, which contradicts our supposition. 
Corollary 4.8 Let the conditions of Theorem 4.7 be fulfilled, and let the set $M_{x}$ be connected for any $x \in \Omega$. Then, for each $M_{x}$, equation (2.1) has exactly one solution $f \in C\left(M_{x}, B\right)$.

Proof It is enough to apply Theorem 4.7 for $\Omega^{*}=M_{x}$.

In our consideration below we will assume that $B=\mathbb{R}$, and we denote by $V^{+}$the cone

$$
V^{+}=\{f \in C(\Omega, R) \mid f(x) \geq 0, x \in \Omega\} .
$$

Theorem 4.9 Let the following conditions be fulfilled:

1. The metric space $\Omega$ is connected and $0<\mu(\Omega) \leq \infty$.

2. Conditions (A), (AM), (S1), and (S4) hold, and for any $x \in \Omega$ the set $M_{x}$ is connected.

3. For each $x \in \Omega$, there exists an $M$-star continuum $\Omega_{x} \subset \Omega$ with $\mu\left(\Omega_{x}\right)<\infty$ such that $M_{x} \subset \Omega_{x}$.

4. For arbitrary $x, y \in \Omega$, the operator $Q$ is monotonic in $V^{+}$with regard to the order induced by the cone $V^{+}$.

Then, for each $p \in C(\Omega, R)$ and every $x \in \Omega$, equation (2.1) has a unique solution $f \in$ $C\left(\Omega_{x}, R\right)$ in $\Omega_{x}$.

Proof Let $x \in \Omega$ be an arbitrary point and $\Omega_{x} \subset \Omega$ be the $M$-star continuum existing according to condition 3 of Theorem 4.9. Lemma 4.1 implies that the operator $K$ defined by (4.1) maps $C\left(\Omega_{x}, R\right)$ into $C\left(\Omega_{x}, R\right)$. Then, from (4.1) for each $s \in \Omega_{x} \cup\{x\}$ and $f \in C\left(\Omega_{x}, R\right)$, we have

$$
K f(s)=p(s)+\int_{M_{s}}(Q(s, y, f(y))-Q(s, y, 0)+Q(s, y, 0)) d \mu_{y} .
$$

Define $h(s)=|p(s)|+\int_{M_{s}}|Q(s, y, 0)| d \mu_{y}$ and define for every $f \in C\left(\Omega_{x}, R\right)$ the linear continuous and positive operator $L f(s)=L\left(\Omega_{x}\right) \int_{M_{s}} f(y) d \mu_{y}$ for $s \in \Omega_{x} \cup\{x\}$. From Theorem 2 of [8] it follows that the spectral radius of the operator $L$ is equal to zero. Therefore the equation $L f(s)+h(s)=f(s)$ has a unique solution $f_{0} \in V^{+}$. For every $f \in V^{+}$, from (4.7) and condition (S4) it follows that $K f(s) \leq L f(s)+h(s)$ for each $s \in \Omega_{x} \cup\{x\}$. Then the operator $K$ maps the order interval

$$
\left[0, f_{0}\right]=\left\{f \in C\left(\Omega_{x}, R\right) \mid 0 \leq f(s) \leq f_{0}(s), s \in \Omega_{x} \cup\{x\}\right\}
$$

into itself, and therefore $K$ has at least one fixed point in the interval (see [20], Chapter 8).

From Theorem 4.7 it follows that for each $x, y \in \Omega$ for which $M_{x} \cap M_{y} \neq \varnothing$ and for any solutions $f_{x}$ and $f_{y}$, we have that $f_{x}(s)=f_{y}(s)$ for $s \in M_{x} \cap M_{y}$, and therefore the global solution $f_{\Omega}$ defined with (2.1) for all $x \in \Omega$ is unique. Note that the solution $f_{\Omega}$ is continuous in $\Omega_{x}$ for each $x \in \Omega$.

It is easy to see that if $\Omega$ is a local compact metric space, then the solution $f_{\Omega}$ is continuous in $\Omega$, i.e., $f_{\Omega} \in C(\Omega, R)$.

\section{Inequalities}

In this section we apply the results obtained in Section 4 to study inequality (2.2). 
Definition 5.1 We say that inequality (2.2) has a local solution in some $M$-star set $\Omega_{*} \subset \Omega$ for some $p \in B C\left(\Omega_{*}, B\right)$ if there exist a point $x_{p} \in \Omega_{*}$ and a function $g \in C\left(M_{x_{p}}, B\right)$ for which $\mu\left(M_{x_{p}}\right)>0$ and $g$ satisfies inequality (2.2) for each $s \in M_{x_{p}}$. If $p, g \in C\left(\Omega_{*}, B\right)$ and $g$ satisfies inequality (2.2) for each $x \in \Omega_{*}$, then we say that $g$ is a solution of (2.2) in $\Omega_{*} \subset \Omega$.

Theorem 5.2 Let the following conditions be fulfilled:

1. $\Omega$ is a connected metric space and $0<\mu(\Omega) \leq \infty$.

2. For each $x \in \Omega$, the sets $M_{x}$ are connected.

3. Conditions (A) hold.

4. The function $Q: \Omega \times \Omega \times \mathbb{R} \rightarrow \mathbb{R}$ for every two fixed elements $x \in \Omega$ and $y \in M_{x}$ is a monotonously increasing function of $v$.

Then, iffor the functions $f, g \in C(\Omega, \mathbb{R})$ the inequality

$$
g(x)-K g(x)<f(x)-K f(x)
$$

holds for each $x \in \Omega$, then $g(x)<f(x)$ for $x \in \Omega$.

Proof Denote by $W$ the following subset of $\Omega$ :

$$
W=\left\{x \in \Omega \mid g(y)<f(y) \text { for each } y \in M_{x}\right\} .
$$

Condition (A4) implies that there exists $x_{0} \in \Omega$ such that $\mu\left(M_{x_{0}}\right)=0$. Then, according to condition (A2), for each $x \in M_{x_{0}}$, we have that $\mu\left(M_{x}\right)=0$. Since $K f(x)=K g(x)$ for each $x \in M_{x_{0}}$, then (5.1) implies that $x_{0} \in W$ and therefore $W \neq \varnothing$.

Let $\left\{x_{n}\right\}_{n=1}^{\infty} \subset W$ be an arbitrary convergent sequence and define $x_{0}=\lim _{n \rightarrow \infty} x_{n}$. If we assume that $x_{0} \notin W$, then there exists a point $y \in M_{x_{0}}$ such that $g(y) \geq f(y)$, and therefore from (5.1) it follows that

$$
K g(y)-K f(y)>0
$$

Then there exists a set $G \subseteq M_{y}$ such that $\mu(G)>0$ and $g(s)>f(s)$ for each $s \in G$. Then, for each $n \geq 1$, we have that $\mu(G) \leq \mu\left(M_{y} \backslash M_{x_{n}}\right) \leq \mu\left(M_{x} \Delta M_{x_{n}}\right)$ and from condition (A2) it follows that $\mu(G)=0$, which contradicts our assumption. Thus we prove that $W$ is a closed set.

It is easy to see that if $x \in W$, then obviously $M_{x} \subset W$, i.e., $W$ is an $M$-star set. Since $f, g \in C(\Omega, \mathbb{R})$, then for each $y \in M_{x}, x \in W$, there exists an open ball $U\left(y, \varepsilon_{y}\right)$ such that $U\left(y, \varepsilon_{y}\right) \subset W$. Then the set $U_{x}=\bigcup_{y \in M_{x}} U\left(y, \varepsilon_{y}\right)$ is an open cover of $M_{x}$ and $U_{x} \subset W$. Since $M_{x}$ is compact, there exists a finite number of balls such that $\bigcup_{k=1}^{n} U\left(y_{k}, \varepsilon_{y_{k}}\right) \subset W$. Then, according to Theorem 3.19, there exists $\delta\left(\varepsilon_{y_{1}}, \ldots, \varepsilon_{y_{n}}\right)>0$ such that for each $s \in \Omega, \rho(s, x)<$ $\delta$ we have that $M_{s} \subset W$, and therefore we prove that $W$ is an open set.

Since $\Omega$ is a connected metric space, then we can conclude that $W=\Omega$. Then we have that for each $x \in \Omega$ and $y \in M_{x}$, the following inequality $Q(x, y, g(y))<Q(x, y, f(y))$ holds. Then from (5.1) it follows that

$$
g(x)<g(x)-K g(x)+K g(x)<f(x)-K f(x)+K g(x),
$$

and therefore $g(x)<f(x)$ for each $x \in \Omega$. 
Remark 5.3 It is easy to see that if we replace the strict inequality (5.1) by a non-strict one, the assertion of the theorem still holds for every $x \in \Omega \backslash \operatorname{Ker} M$.

Corollary 5.4 Let the following conditions be fulfilled:

1. Conditions of Theorem 4.9 hold.

2. Condition 4 of Theorem 5.2 holds.

Then, for every solution $g \in C(\Omega, \mathbb{R})$ of inequality (2.2) and for every $x \in \Omega$, the inequality $g(x) \leq f(x)$ holds, where $f \in C(\Omega, \mathbb{R})$ is the unique solution of equation (2.1).

Proof According to Theorem 4.9, equation (2.1) has a unique solution $f \in C(\Omega, \mathbb{R})$. For every solution $g \in C(\Omega, \mathbb{R})$ of inequality (2.2), we have that the inequality

$$
g(x)-K g(x) \leq f(x)-K f(x)
$$

holds for every $x \in \Omega$. Then if for some $x \in \Omega$ we have that

$$
g(x)-K g(x)=f(x)-K f(x),
$$

then we can conclude that $g(x)=f(x)$ ( $f$ is the unique solution of equation (2.1)). For other points $x \in \Omega$ for which inequality (5.2) is strict, the assertion of Corollary 5.4 follows from Theorem 5.2.

\section{Applications}

As an illustration of the results obtained in the previous sections, we will consider integral inequalities with maxima.

Let $\Omega=\mathbb{R}_{+}^{2}=[0, \infty) \times[0, \infty), \rho$ is the Euclidean metric, $B=\mathbb{R}, \mu$ is the Lebesgue measure, $c, a, b>0$ are arbitrary constants, $x=\left(x_{1}, x_{2}\right) \in \mathbb{R}^{2}$ and define $T_{x}=\left[x_{1}-a, x_{1}\right] \times\left[x_{2}-\right.$ $\left.b, x_{2}\right]$ and $I(a, b)=\{[-a, \infty) \times[-b, \infty)\} \backslash \mathbb{R}_{+}^{2}$. The function $k: \mathbb{R}_{+}^{2} \rightarrow[0, \infty)$ is continuous and the operator $Q: \Omega^{2} \times \mathbb{R} \rightarrow \mathbb{R}$ is defined by the following inequality $Q(x, y, f(y))=$ $k(x, y) \max _{s \in T_{y}} f(s)$. Let, for the map $M: \Omega \rightarrow 2^{\Omega}$, conditions (A) and (AM) hold, and consider equation (2.1) and inequality (2.2) under the following conditions:

$$
\begin{aligned}
& f(x)=c+\int_{M_{x}} k(x, y) \max _{s \in T_{y}} f(s) d \mu_{y}, \\
& g(x) \leq c+\int_{M_{x}} k(x, y) \max _{s \in T_{y}} g(s) d \mu_{y}
\end{aligned}
$$

for $x \in \mathbb{R}^{+}$and $g(x) \leq f(x) \leq c$ for $x \in I(a, b)$. It is simple to verify that all conditions of Corollary 5.4 are fulfilled and therefore we have that $g(x) \leq f(x)$ for $x \in \mathbb{R}^{+}$, where $f(x)$ is the unique solution of (6.1) and $g(x)$ is an arbitrary solution of (6.2).

Let us define $\varphi(x)=\max _{s \in T_{y}} f(s), y \in \mathbb{R}^{+}, k(x, y) \equiv k(y)$, and consider the equation

$$
\varphi(x)=c+\int_{M_{x}} k(y) \varphi(y) d \mu_{y}
$$

and $\varphi(x)=c$ for $x \in I(a, b)$. Then from the results in [8] it follows that the equation has a unique solution for which the estimation

$$
g(x) \leq f(x) \leq \varphi(x) \leq c \phi(x)
$$


for $x \in \mathbb{R}^{+}$holds, where $\phi(x)$ is the solution of equation (6.3) when $c=1$, and it can be represented as a convergent Neumann series

$$
\phi(x)=1+\int_{M_{x}} k(y) d \mu_{y}+\int_{M_{x}} k(y)\left(\int_{M_{y}} k\left(y_{1}\right) d \mu_{y_{1}}\right) d \mu_{y}+\cdots
$$

Example Let $\Omega=(0, \infty) \times(0, \infty), B=\mathbb{R}$ and $M_{x}=\left[0, x_{1}\right] \times\left[0, x_{2}\right]$. Then

$$
\phi(x)=\exp \left(\int_{0}^{x_{1}}\left(\int_{0}^{x_{2}} k(y) d y_{2}\right) d y_{1}\right)
$$

\section{Competing interests}

The authors declare that they have no competing interests.

\section{Authors' contributions}

All authors contributed equally to the writing of this paper. All authors read and approved the final manuscript.

\section{Acknowledgements}

This paper is partially supported by Plovdiv University NPD grant NI13 FMI-002.

Received: 10 December 2013 Accepted: 20 June 2014 Published: 22 Jul 2014

\section{References}

1. Agarwal, RP: Difference Equations and Inequalities: Theory, Methods and Applications, 2nd edn. Pure and Applied Mathematics, vol. 228. Dekker, New York (2000)

2. Agarwal, RP, O'Regan, D, Wong, PJY: Constant-Sign Solutions of Systems of Integral Equations. Springer, Berlin (2013)

3. Pachpatte, BG: Multidimensional Integral Equations and Inequalities. Atlantis Studies in Mathematics for Engineering and Science, vol. 9. Atlantis Press, Paris (2011)

4. Dragomir, SS: Some Gronwall Type Inequalities and Applications. Nova Science Publishers, New York (2003)

5. Agarwal, RP, O'Regan, D: Infinite Interval Problems for Differential, Difference, and Integral Equations. Kluwer Academic, Dordrecht (2001)

6. Agarwal, RP, Ding, S, Nolder, C: Inequalities for Differential Forms. Springer, New York (2009)

7. Appell, JM, Kalitvin, AS, Zabrejko, PP: Partial Integral Operators and Integro-Differential Equations. Monographs and Textbooks in Pure and Applied Mathematics. Dekker, New York (2000)

8. Bainov, DD, Myshkis, AD, Zahariev, Al: On an abstract analog of the Bellman-Gronwall inequality. Publ. Res. Inst. Math. Sci. 20(5), 903-911 (1984)

9. Magnucka-Blandzi, E, Popenda, J, Agarwal, RP: Best possible Gronwall inequalities. Math. Comput. Model. 26(3), 1-8 (1997)

10. Zahariev, Al, Bainov, DD: A note on Bellman-Gronwall's inequality. J. Math. Anal. Appl. 1, 147-149 (1981)

11. Guryanova, IE, Myshkis, AD: Nonextendable solutions of abstract Volterra type integral equations. Differ. Equ. 22(10), 1786-1789 (1986) (in Russian)

12. Ronkov, A, Bainov, D: Integral equations and inequalities of Volterra type for functions defined in partially ordered spaces. J. Math. Anal. Appl. 125, 483-507 (1987)

13. Ronkov, A, Bainov, D: Nonlinear integral inequalities for functions defined in partially ordered topological spaces. Nonlinear Anal. TMA 11(3), 297-304 (1987)

14. Bainov, DD, Kostadinov, SI, Zahariev, Al: Abstract Volterra type integral equations. Bull. Inst. Math. Acad. Sin. 16(1), 93-104 (1988)

15. Drakhlin, ME, Litsyn, E: Volterra operator: back to the future. J. Nonlinear Convex Anal. 6(3), 375-391 (2005)

16. Litsyn, E: Representation formula for solution of a functional equation with Volterra operator. J. Math. Anal. Appl. 336, 1073-1089 (2007)

17. Hille, E, Phillips, R: Functional Analysis and Semi-Groups. American Mathematical Society Colloquium Publications. Am. Math. Soc., Providence (1957)

18. Edwards, RE: Functional Analysis. Holt, Rinehart \& Winston, New York (1965)

19. Dugundji, J: An extension of Tietze's theorem. Pac. J. Math. 1, 353-367 (1951)

20. Hutson, VCL, Pym, JS: Application of Functional Analysis and Operator Theory. Academic Press, London (1980)

10.1186/1029-242X-2014-260

Cite this article as: Zahariev et al.: On Volterra-type integral equations in noncompact metric space. Journal of Inequalities and Applications 2014, 2014:260 